\title{
Temperature Dependence of Epitaxial Graphene Formation on $\operatorname{SiC}(0001)$
}

\author{
LUXMI, ${ }^{1}$ SHU NIE,${ }^{1}$ P.J. FISHER,${ }^{1}$ R.M. FEENSTRA,${ }^{1,4}$ GONG GU ${ }^{2}$ and \\ YUGANG SUN ${ }^{3}$
}

\begin{abstract}
1.-Department of Physics, Carnegie Mellon University, Pittsburgh, PA 15213, USA. 2.-Sarnoff Corporation, CN5300, Princeton, NJ 08543, USA. 3.-Center for Nanoscale Materials, Argonne National Laboratory, Argonne, IL 60439, USA. 4.-e-mail: feenstra@cmu.edu
\end{abstract}

The formation of epitaxial graphene on $\mathrm{SiC}(0001)$ surfaces is studied using atomic force microscopy, Auger electron spectroscopy, electron diffraction, Raman spectroscopy, and electrical measurements. Starting from hydrogenannealed surfaces, graphene formation by vacuum annealing is observed to begin at about $1150^{\circ} \mathrm{C}$, with the overall step-terrace arrangement of the surface being preserved but with significant roughness (pit formation) on the terraces. At higher temperatures near $1250^{\circ} \mathrm{C}$, the step morphology changes, with the terraces becoming more compact. At $1350^{\circ} \mathrm{C}$ and above, the surface morphology changes into relatively large flat terraces separated by step bunches. Features believed to arise from grain boundaries in the graphene are resolved on the terraces, as are fainter features attributed to atoms at the buried graphene/SiC interface.

Key words: Graphene, silicon carbide, semiconductor, field-effect transistor

\section{INTRODUCTION}

Graphene (one or more monolayers of carbon) has been intensively studied for the past few years because of its unique electrical behavior. Graphene exists in two main forms: isolated layers formed by exfoliation of graphite, ${ }^{1}$ and epitaxial layers residing on a suitable lattice-matched substrate. ${ }^{2}$ The size of the graphene flakes formed by the exfoliation process is relatively small, so many workers have focused on the epitaxial approach for obtaining films suitable for large-scale fabrication of circuits. There are several methods for forming epitaxial graphene, with the most studied to date being the sublimation of silicon from $\mathrm{SiC}$ leaving behind excess carbon in the form of graphene. $^{2}$ Field-effect transistors fabricated on epitaxial graphene/SiC have yielded room-temperature field-effect mobilities of $5000 \mathrm{~cm}^{2} / \mathrm{V}$ s or more. ${ }^{3,4}$

In this work, we produce graphene by sublimation of $\mathrm{Si}$ from $\mathrm{SiC}(0001)$ (i.e., the so-called Si-face of

(Received August 21, 2008; accepted September 30, 2008; published online October 21, 2008)
$\mathrm{SiC})$, using the well-known procedure of heating the $\mathrm{SiC}$ in vacuum. Use of semi-insulating $\mathrm{SiC}$ precludes heating by direct current, and a metal film (which would allow electron-beam heating) cannot be deposited on the backside of the wafer since this metal is found to migrate to the front of the wafer during heating. ${ }^{5}$ Furthermore, poor thermal contact between sample and heater (due to the vacuum environment) and low optical absorption of the $\mathrm{SiC}$ (band gap $\approx 3.0 \mathrm{eV}$, depending on polytype) necessitates temperatures as high as $1850^{\circ} \mathrm{C}$ for the heater itself. To accomplish this heating we have developed a simple arrangement consisting of a graphite strip, with currents as high as 200 A passing through the strip. Prior to the graphene formation, the substrates are hydrogen-etched at $\approx 1600^{\circ} \mathrm{C}$ in order to remove residual polishing damage. The graphite strips are found to be quite robust in this environment, unlike other heater materials that we have tested.

We have investigated the formation of graphene using annealing temperatures ranging from $1100^{\circ} \mathrm{C}$ to $1500^{\circ} \mathrm{C}$, and characterized our samples using 
atomic force microscopy (AFM), Auger electron spectroscopy (AES), low-energy electron diffraction (LEED), Raman spectroscopy, and electrical measurements. The evolution of the morphology is studied in particular, revealing motion of step edges, pit formation, and subsequent coarsening on the surface, and features associated with grain boundaries in the graphene as well as structure of the graphene/SiC interface.

\section{EXPERIMENTAL}

The graphite strip heater we use is contained in a dedicated ultra-high-vacuum (UHV) chamber with a base pressure of $1 \times 10^{-10}$ Torr, pumped by a $150 \mathrm{l} / \mathrm{s}$ turbo-molecular pump and a hydrogen-getter pump. A graphite plate with a thickness of $1 \mathrm{~mm}$ and an area of $100 \mathrm{~mm} \times 75 \mathrm{~mm}$ is cut into a bow-tie shape, with a narrow neck of $20 \mathrm{~mm}$ length and $14 \mathrm{~mm}$ width. Two thick (dual, $9.5 \mathrm{~mm}$ diameter) watercooled copper feedthroughs are used to transmit the current, mounted onto large copper clamps on the two 75-mm ends of the plate. The current is supplied by a transformer capable of supplying up to $210 \mathrm{~A}$ at $6.3 \mathrm{~V}$. Gate valves separate the turbo pump from its backing pump as well as the hydrogen-getter pump from the main chamber; these gate valves are closed and the turbo-pump is switched off for the H-etching, and they are open with the turbo-pump switched on for the graphitization.

Most of our experiments have been performed on nominally on-axis, semi-insulating $4 \mathrm{H}-\mathrm{SiC}$ substrates that were purchased from Cree Corp. As received, these substrates had been mechanically polished on both sides and they are epi-ready (i.e., with further polishing and a damage removal step) on the (0001) surface. Samples measuring $10 \mathrm{~mm} \times$ $10 \mathrm{~mm}$ were cut from the wafers. Hydrogen-etching was performed at $1 \mathrm{~atm}$ pressure, using $99.9995 \%$ purity hydrogen with a flow rate of $10 \mathrm{lpm}$ and at a temperature of $1550^{\circ} \mathrm{C}$ for $3 \mathrm{~min}$ to eliminate scratches. Temperature is measured with a disappearing filament pyrometer; the pyrometer is directed at the sample, although since the sample is transparent it is mainly the heater strip that is seen. The turbo-pump is restarted a few minutes after the $\mathrm{H}$-etching and the gate valve to the $\mathrm{H}$-getter pump is opened shortly thereafter. The pressure reaches $1 \times 10^{-8}$ Torr after pumping for about $30 \mathrm{~min}$, and the annealing to form the graphene is then performed. All results refer to the surface of the sample that is facing away from the heater strip.

The material used to fabricate the graphite heater strip was obtained from Poco Graphite, and is semiconductor-grade material. No measurable contamination as seen by residual gas analysis is found to be emitted during the graphitization (these measurements were performed only after the first few heating runs with the strip). The strip is found to be quite robust; we have processed $>50$ samples with it and it shows only a small amount of pitting on the surface as a result. In contrast, we have previously used thin $(25 \mu \mathrm{m})$ Ta foils for the $\mathrm{H}$-etching and they are found to disintegrate after each H-etching run, presumably due to embrittlement by $\mathrm{H}$ uptake. We also attempted the use of $\mathrm{SiC}$ heating strips, but they were found to be relatively brittle and cracked after one or two runs.

The thickness (number of graphene monolayers) of our graphene films is determined by AES, using $5-\mathrm{kV}$ incident electrons and a VG Scientific Clam 100 hemispherical analyzer. For calibration, we use a spectrum obtained from the $\operatorname{SiC}(0001) \sqrt{3} \times \sqrt{3}$ R30 surface as shown in Fig. 1. This surface has a known structure of Si adatoms sitting on top of a $\mathrm{SiC}$ bilayer with one adatom for each three $\mathrm{SiC}$ unit cells. ${ }^{6}$ We analyze the intensities of the C KLL line at $272 \mathrm{eV}$ to the Si LMM line at $92 \mathrm{eV}$ by summing the electron emission from consecutive layers with a phenomenological form for the electron escape depth, ${ }^{7}$ and we determine a ratio of C-to-Si sensitivity factors with this measurement. We then apply a similar model to spectra obtained from the graphene on $\mathrm{SiC}$. For this purpose we assume a model of uniform monolayers of graphene $(\mathrm{ML}=$ 38.0 carbon atoms $/ \mathrm{nm}^{-2}$ ) on the SiC, with $0.335 \mathrm{~nm}$ spacing between graphene layers and also between the uppermost SiC layer and the first graphene layer (which is actually a graphene-like buffer layer-see below). The resulting relationship between Auger C-to-Si ratio and number of carbon layers is shown in Fig. 1. Our results are consistent with the prior work of de Heer et al., ${ }^{2}$ except for details of the interface structure between graphene and SiC. An interface phase is known to exist,

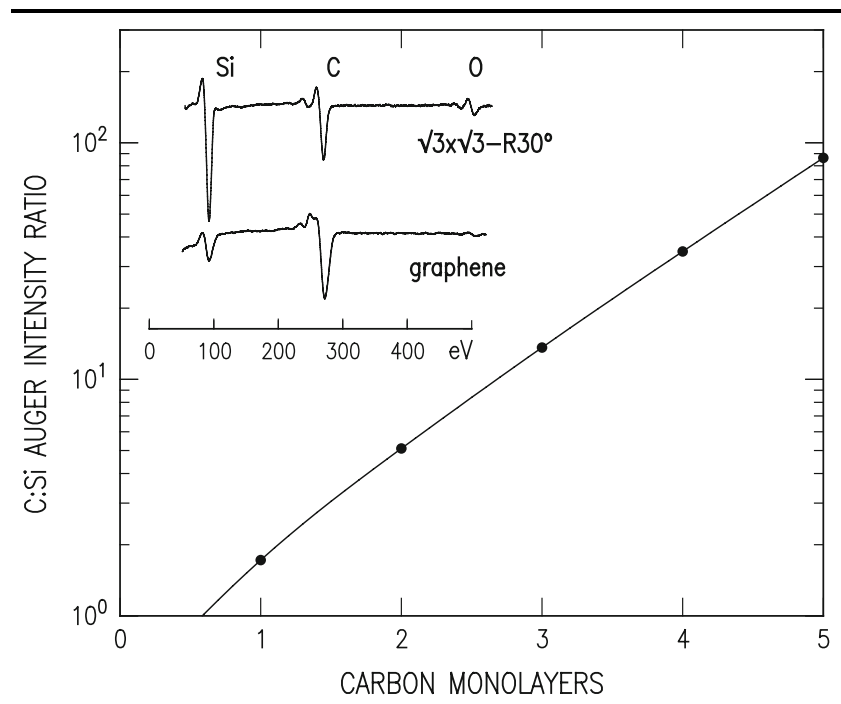

Fig. 1. Calibration curve relating $\mathrm{C}$-to-Si Auger intensity ratio to the number of excess carbon monolayers (relative to a SiC bilayer) on the surface. A model consisting of graphene layers uniformly spaced at $0.335 \mathrm{~nm}$ from the interface is assumed. The inset shows Auger electron spectra acquired from a UHV-prepared $\mathrm{SiC}(0001) \sqrt{3} \times \sqrt{3}$ $\mathrm{R} 30^{\circ}$ surface, and from a graphitized surface prepared by annealing at $1150^{\circ} \mathrm{C}$ for $40 \mathrm{~min}$. 
namely, the bare (i.e., not covered with graphene) $6 \sqrt{3} \times 6 \sqrt{ } 3-\mathrm{R} 30^{\circ}$ reconstruction, ${ }^{8,9}$ which has recently been found to contain close to one graphene ML of excess carbon relative to surface terminated with a SiC bilayer. ${ }^{10}$ We can therefore estimate the number of graphene layers to be simply one fewer than the number of carbon layers.

Raman spectra were measured on a Raman microscope (Renishaw, inVia) with excitation wavelength of $514 \mathrm{~nm}$. All spectra were measured using a $100 \times$ microscope objective to focus the laser excitation $(10 \mathrm{~mW})$ onto the samples as well as to collect the scattered light. The measurements were performed at room temperature.

\section{RESULTS AND DISCUSSION}

\section{Structure}

Figure 2 shows the temperature dependence of the thickness of our graphene films. In this plot, and throughout the remainder of this paper, we refer to the number of graphene monolayers, which, as described in the previous section, is one fewer than the number of carbon monolayers. ${ }^{10}$ We first examine the data points in Fig. 2a shown by open circles, for which the annealing temperatures are obtained by viewing the sample directly with the pyrometer. Since the sample is transparent, the

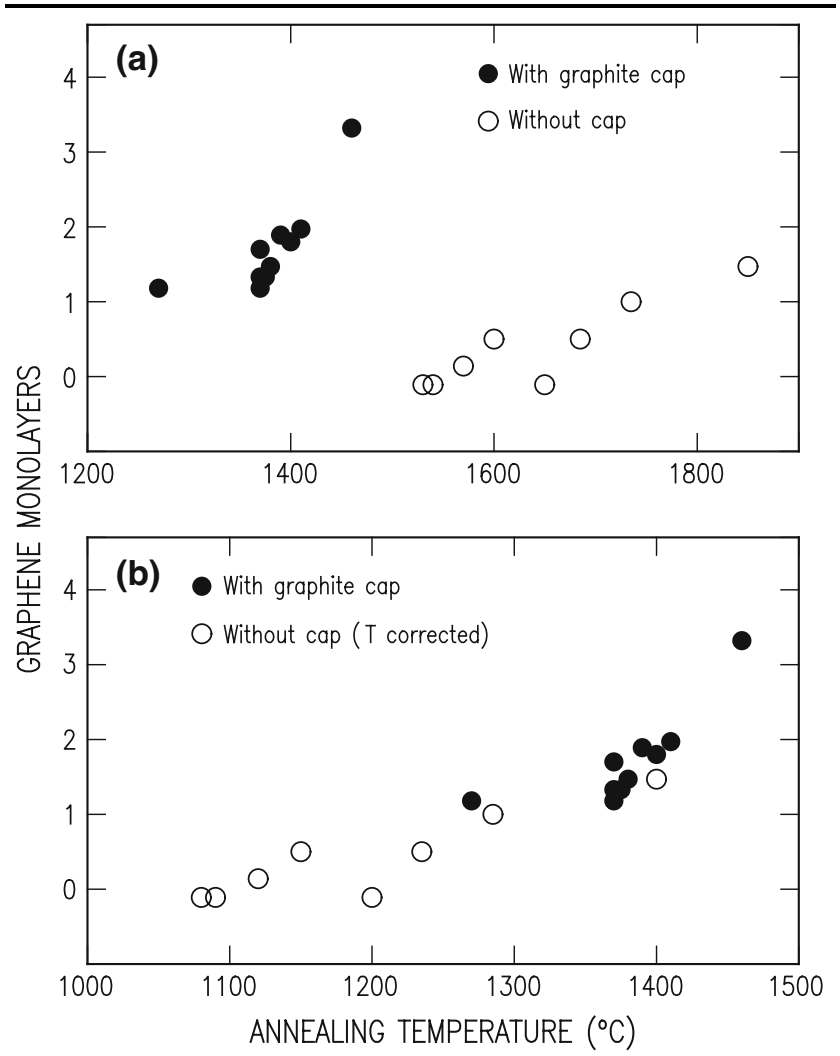

Fig. 2. Graphene thickness as a function of annealing temperature: (a) using raw pyrometry data for the temperatures, and (b) using a $450^{\circ} \mathrm{C}$ temperature correction for points measured without the graphite cap in place. Annealing time is $\mathbf{4 0} \mathrm{min}$ for all data points. pyrometer sees mainly the heater in this case. We find that $\geq 1 \mathrm{ML}$ of graphene is formed only for the highest temperature used, $1850^{\circ} \mathrm{C}$. This value is significantly higher than the $\approx 1350^{\circ} \mathrm{C}$ reported in previous studies for multilayer graphene films, ${ }^{2,4,5}$ and we conclude that a large discrepancy is occurring between the heater temperature and the actual sample temperature, presumably because of poor thermal contact between sample and heater.

To improve our determination of sample temperature, we use a small graphite "cap" piece that sits on top of the sample. This cap, fabricated from 1-mm-thick graphite, measures about $13 \mathrm{~mm} \times$ $13 \mathrm{~mm}$ and has a small depression milled into it so that it fits over the $10 \mathrm{~mm} \times 10 \mathrm{~mm}$ sample. A hole with a diameter of $6.35 \mathrm{~mm}$ is drilled through the center of the cap piece, thus allowing the sample surface over this area to be exposed to the vacuum. With this cap in place, we measure its temperature as well as that of the heater strip, using the pyrometer. For a strip temperature of $2000 \mathrm{~K}$ (i.e., $1727^{\circ} \mathrm{C}$ ), we find a cap temperature that is $315 \pm 15 \mathrm{~K}$ lower than that of the strip. Comparing with expectations from black-body radiation, the bottom of the cap will absorb radiation from the strip (the sample being transparent), and both the bottom and the top will radiate, so balancing the input and output powers we expect a cap temperature to be a factor $0.5^{1 / 4}$ times that of the strip, corresponding to a temperature difference of $318 \mathrm{~K}$. The good agreement with observation indicates that radiation is the dominant heat transfer mechanism. With the cap present, we do not expect the sample temperature to be lower than that of the cap, and we simply use the cap temperature as an estimate of the sample temperature. In the absence of the cap, we estimate the temperature difference between sample and strip simply by shifting the data in Fig. 2a such that data points with and without the cap being present are aligned, as shown in Fig. $2 \mathrm{~b}$. We find that a $450^{\circ} \mathrm{C}$ shift is appropriate, and we use this correction for all data measured without the cap.

The morphology of the graphene film prepared at various annealing temperatures is shown in Fig. 3. Prior to annealing the morphology of the samples revealed a uniform step-terrace array, as previously described. ${ }^{11}$ After annealing at about $1150^{\circ} \mathrm{C}$, the overall step morphology is preserved (steps run approximately vertically in the image) although pits are seen to form in the areas between the steps, as shown in Fig. 3a. This morphology is very similar to what we obtained in our prior experiments, ${ }^{5,12}$ and it has recently been studied by Hannon and Tromp, who provide a detailed formation mechanism for the morphology based on the development of the $6 \sqrt{ } 3 \times 6 \sqrt{3} 3-R 30^{\circ}$ "buffer layer." 10 This $6 \sqrt{ } 3$ layer forms prior to the formation of graphene, and graphene then forms on top of the $6 \sqrt{ } 3$ structure. $^{8-10,13,14}$ At a higher temperature of around $1250^{\circ} \mathrm{C}$, we find motion of the steps such that the 

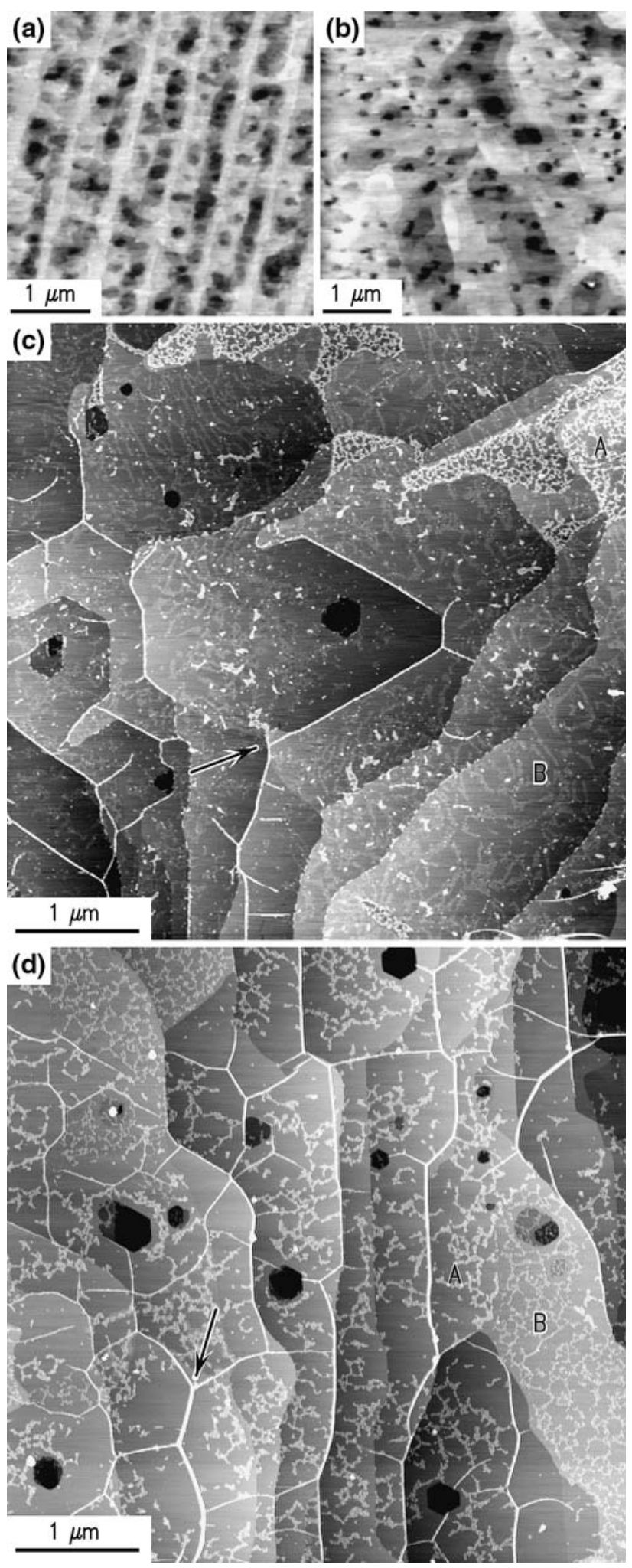

Fig. 3. AFM images of graphene on $\mathrm{SiC}(0001)$ surfaces prepared under various annealing conditions: (a) $1150^{\circ} \mathrm{C}$ for 40 min resulting in a graphene thickness of $0.5 \mathrm{ML}$, (b) $1285^{\circ} \mathrm{C}$ for 40 min resulting in a graphene thickness of $1.0 \mathrm{ML}$, (c) $1370^{\circ} \mathrm{C}$ for 40 min resulting in a graphene thickness of $1.2 \mathrm{ML}$, and (d) $1390^{\circ} \mathrm{C}$ for 40 min resulting in a graphene thickness of $1.9 \mathrm{ML}$. Images are displayed with gray scale ranges of $4 \mathrm{~nm}, 3 \mathrm{~nm}, 2 \mathrm{~nm}$, and $3 \mathrm{~nm}$, respectively. ordered step-terrace array is no longer seen on the surface, as shown in Fig. 3b. The surface pits formed have begun to coarsen here ${ }^{10}$ (with smaller pits combining to form larger ones) and the shapes of the highest terraces tend to be more compact in shape as compared with the extended linear features of Fig. 3a.

At a higher annealing temperature near $1350^{\circ} \mathrm{C}$, the steps undergo considerable motion, forming surface regions microns in size with relatively few steps, separated by narrower regions containing step bunches. Figure 3c and d displays AFM images acquired from the relatively flat surface areas. Two interesting morphological features are seen in these images: the white lines or ridges $(1 \mathrm{~nm}$ to $2 \mathrm{~nm}$ high) occurring both near step edges and on terraces, two of which are marked by arrows in Fig. 3c and $d$, and the finger-like patterns seen on many of the terraces, e.g., as marked by A and B in Fig. 3c and $d$. These fingers form complex fractal-like patterns, well known from the theory of diffusionlimited aggregation (DLA). ${ }^{15}$ Different intensities of the finger-like patterns are seen in different terrace regions, e.g., as marked by $\mathrm{A}$ and $\mathrm{B}$ terraces in each of Fig. 3c and d, suggesting that the structures responsible for the patterns are located at the graphene/SiC interface, with the interface occurring at different depths below the surface for different terraces. Also, near the observed ridges, the fingers tend to be absent from the nearby terrace regions. At higher temperatures above about $1400^{\circ} \mathrm{C}$, we do not discern any of the finger-like patterns on the terraces, presumably because of the thicker films in those cases. Also, for these surfaces, the pits on the surface continue to coarsen and grow in size, and they tend to act as pinning centers for the observed ridges.

Based on these observations, we suggest that the ridges and finger-like patterns both arise from a condensation of mobile atoms that are present at the graphene/SiC interface during the hightemperature annealing. As mentioned above, the interface is known to have the $6 \sqrt{ } 3$ structure, and it is entirely possible that some or all of the atoms in this structure are mobile at the annealing temperature. When the sample is cooled down, these mobile $\mathrm{C}$ or $\mathrm{Si}$ atoms will condense; we propose that the observed lines form when the condensation occurs at a domain boundary within the $6 \sqrt{ } 3$ structure, the graphene, and/or the $\mathrm{SiC}$. Direct evidence for such domain boundaries can be seen in the recent work of Poon et al. ${ }^{16}$ On a surface partially covered with a graphene layer, they observed narrow intervening domains of $6 \sqrt{ } 3$ structure (see their Fig. 2), ${ }^{16}$ and the neighboring graphene layers are translationally inequivalent. We thus associate the observed ridges with domain boundaries in the graphene, formed due to an underlying boundary in the $6 \sqrt{ } 3$ structure. As the sample is cooled, mobile atoms from the interface will condense at these 
(a)

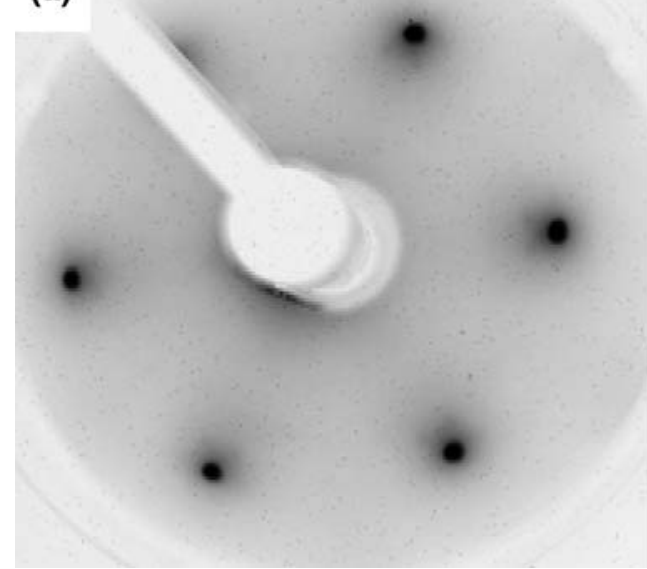

(b)

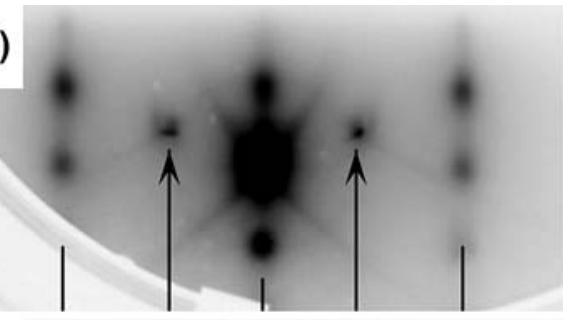

(c)

Fig. 4. (a) LEED pattern acquired at $133 \mathrm{eV}$, of a surface prepared by annealing at $1410^{\circ} \mathrm{C}$ for 40 min resulting in a graphene thickness of 2.0 ML. (b) and (c) RHEED patterns of the same sample as in (a), with electron beam along $\langle 1 \overline{100}\rangle$ and $\langle 11 \overline{2} 0\rangle$ azimuths, respectively. Vertical lines indicate features from the graphene, and arrows show features from the underlying SiC. All diffraction patterns are shown in reverse contrast.

boundaries, or if no boundary is near enough then they homogeneously condense forming a DLA pattern.

Electron diffraction from a film similar to that of Fig. 3d is shown in Fig. 4. In LEED (Fig. 4a) we see simply the hexagonal array of spots expected for the graphene surface. (Detailed evolution of the LEED pattern with graphene thickness has been previously reported by several authors, ${ }^{8,9,13,14}$ and also observed by us. ${ }^{17}$ ). The SiC substrate cannot be discerned in this pattern due to the thickness of the graphene. However, using RHEED we can faintly resolve the substrate, as shown in Fig. 4b. Vertical lines in that image mark the zeroth- and first-order RHEED streaks; their spacing is $\sqrt{ } 3$ times that of Fig. $4 \mathrm{c}$ obtained with a $30^{\circ}$ change in sample orientation, as expected for a hexagonal surface. The arrows in Fig. $4 \mathrm{~b}$ point to faint streaks that are located at a fraction $0.46 \pm 0.01$ of the first-order graphene spacing (the intense dots superimposed on the streaks arise from an intersection of a Kikuchi line with the streak; the sample orientation was carefully adjusted to achieve this intersection in order to enhance the intensity of the streak itself). We expect a fractional spacing of $a_{\mathrm{C}} /\left(a_{\mathrm{SiC}} \sqrt{3}\right)=$ 0.461 using lattice constants of $a_{\mathrm{C}}=0.246 \mathrm{~nm}$ and $a_{\mathrm{SiC}}=0.308 \mathrm{~nm}$, in agreement with experiment.

\section{Raman Spectroscopy}

Typical Raman spectra from our graphene films are shown in Fig. 5. Each spectrum shows the known fingerprint features associated with graphene: The weak D peak at $1357 \mathrm{~cm}^{-1}$ and intense G peak at $1583 \mathrm{~cm}^{-1}$ correspond to graphene zoneedge and zone-center phonons, respectively (the D peak is normally forbidden, and its presence indicates disorder in the film). ${ }^{18,19}$ The intense 2D peak located at $2706 \mathrm{~cm}^{-1}$ is attributed to the doubly resonant scattering from the zone-edge phonons. ${ }^{18,19}$

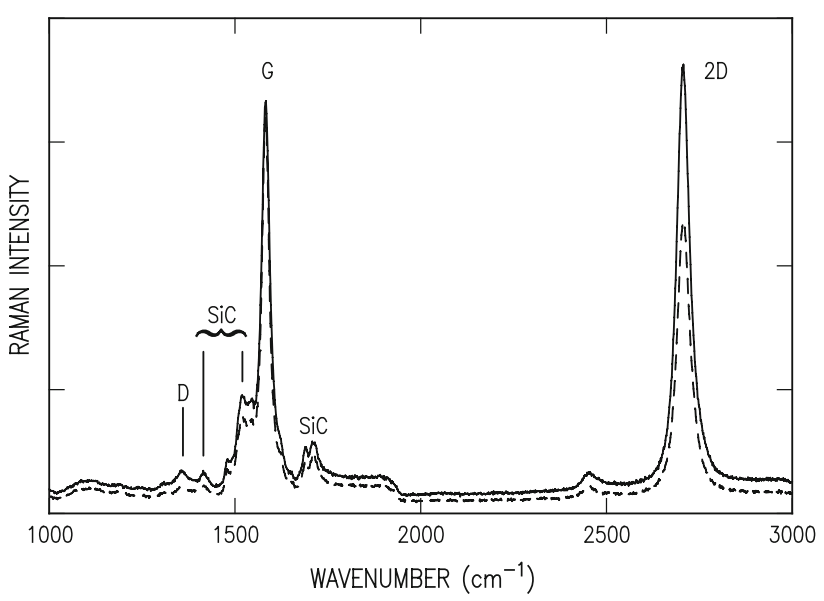

Fig. 5. Raman spectra acquired from a sample annealed at $1370^{\circ} \mathrm{C}$ for $40 \mathrm{~min}$, yielding 1.7 ML of graphene. Spectra acquired from two different locations on the sample are shown.

The weak peak at $2453 \mathrm{~cm}^{-1}$ also arises in some way from the graphene. ${ }^{18,19}$ Spectral features from the SiC substrate are also observed in each spectrum: The peaks at $1519 \mathrm{~cm}^{-1}, 1690 \mathrm{~cm}^{-1}$, and $1710 \mathrm{~cm}^{-1}$ (as well as features below the observed G peak) arise from double resonance of $\mathrm{SiC}$ optical phonons. ${ }^{20}$ The weak peak at $1415 \mathrm{~cm}^{-1}$ is not associated with bulk phonons of $\mathrm{SiC}$, but it nevertheless does arise from the substrate since we find its intensity to increase as the focus of the probing laser is moved away from the surface and into the substrate. This peak is not seen for all the $\mathrm{SiC}$ substrates we have studied, and we tentatively attribute it to some sort of defect in this particular substrate.

The positions of the graphene peaks are found to be quite uniform over the surface, but significant variation in peak intensities do occur, as seen by comparing the two spectra of Fig. 5. We tentatively attribute these variations to varying thickness of the graphene film. Graf et al. have recently 
quantified such intensity variations in a study of exfoliated graphene, in which they find that the ratio of integrated intensities of the G-peak and 2D-peak can be used to determine the thickness. ${ }^{21}$ We find this ratio to be 0.20 and 0.29 for the spectra shown by solid and dashed lines, respectively, in Fig. 5 (we have removed the contributions of the $\mathrm{SiC}$ optical-phonon band from the $\mathrm{G}$ peak using a spectrum obtained from bare $\mathrm{SiC}$, and we use a Gaussian fit for the $\mathrm{G}$ peak and a Lorentzian for the $2 \mathrm{D}$ peak). Using the relationship between intensity ratio and thickness provided by Graf et al., ${ }^{21}$ we find that these ratios correspond to graphene thicknesses of 1.3 ML and 1.6 ML, respectively. The average of these results, $1.45 \mathrm{ML}$, is in fair agreement with the thickness of $1.7 \mathrm{ML}$ derived from our AES model.

\section{Electrical Properties}

The electrical conductance of our graphene films has been measured, simply by measuring the resistance between two probes (bent copper wires) gently

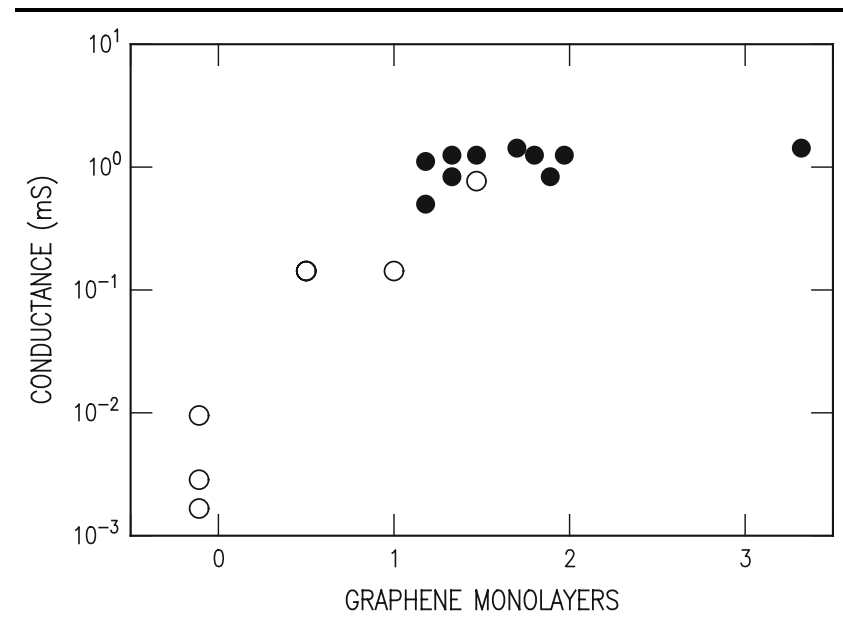

Fig. 6. Conductance of graphene layers (measured with two probes across a $10 \mathrm{~mm} \times 10 \mathrm{~mm}$ sample), as a function of graphene layer thickness. Open and closed symbols have the same meaning as in Fig. 2. placed on two corners of the sample. Results are shown in Fig. 6. We find a sharp onset in the conductance occurring at a graphene thickness of 1.0 ML, with conductance values of about $1(\mathrm{kohm})^{-1}$ for thicker films. In the range of $0 \mathrm{ML}$ to $1 \mathrm{ML}$ the conductance is an order of magnitude smaller than this. For this thickness range, the $6 \sqrt{ } 3$ layer has already formed on the surface, and this structure could be contributing to the conduction. From tunneling spectroscopy, this layer is known to have a narrow band gap of about $300 \mathrm{meV},{ }^{22}$ although at room temperature a nonzero conductivity at the Fermi level is found (likely due to thermal occupation of states across the gap). ${ }^{23}$ In any case, with the complete formation of the first graphene layer the conductivity is significantly increased.

The above simple measurement shows the trend of overall conductance over the $10 \mathrm{~mm} \times$ $10 \mathrm{~mm}$ sample with increasing graphene thickness. Field-effect transistors (FETs) were fabricated as described elsewhere ${ }^{5}$ to measure the local conductance and field-effect mobility of the graphene. Representative results are listed in Table I. All FET measurements were performed at room temperature. Only electron mobilities are listed since most devices are unipolar $n$-channel FETs in the measured gate bias range.

For our samples, two regions with different conductivities are present, and a positive correlation between the mobility and the conductivity is observed. This long-range nonuniformity may be attributed to substrate temperature nonuniformity and/or graphene thickness variation. It is noticed that the graphene conductivity for FETs with relatively high mobility is much higher than in other work $^{3,4}$ and our earlier work, ${ }^{5}$ possibly due to unintentional doping introduced by impurities from the graphite strip heater. Moreover, most FETs are unipolar $n$-channel devices with linear transfer characteristics in the measured gate voltage range $(-100 \mathrm{~V}$ to $+100 \mathrm{~V})$, indicating heavy $n$-type doping; only several FETs exhibit either $p$-type behavior (with hole mobilities $\lesssim 20 \mathrm{~cm}^{2} / \mathrm{V} \mathrm{s}$ ) or flat regions in the transfer characteristics such as that shown by Wu et al. ${ }^{4}$

Table I. FET Measurement Results

\begin{tabular}{|c|c|c|c|c|c|}
\hline $\begin{array}{l}\text { Sample } \\
\text { ID }\end{array}$ & $\begin{array}{c}\text { Annealing } \\
\text { Temperature }\left({ }^{\circ} \mathrm{C}\right)\end{array}$ & $\begin{array}{c}\text { C-to-Si } \\
\text { AES Ratio }\end{array}$ & $\begin{array}{c}\text { Graphene } \\
\text { Thickness (ML) }\end{array}$ & $\begin{array}{l}\text { Conductivity } \\
\qquad(\mathrm{mS})^{\mathrm{a}}\end{array}$ & $\begin{array}{c}\text { Electron } \\
\text { mobility }\left(\mathrm{cm}^{2} / \mathrm{V} \mathrm{s}\right)\end{array}$ \\
\hline 0448.10 & 1370 & 6 & 1.2 & $\begin{array}{c}1.0-1.8 \\
0.29-0.45\end{array}$ & $\begin{array}{c}114-534 \\
34-46\end{array}$ \\
\hline 0448.16 & 1370 & 7 & 1.3 & $\begin{array}{r}1.5-2.8 \\
0.71-1.1\end{array}$ & $\begin{array}{r}169-439 \\
67-218\end{array}$ \\
\hline 0448.14 & 1410 & 13 & 2.0 & $\begin{array}{c}1.3-1.9 \\
0.34-0.99\end{array}$ & $\begin{array}{l}70-333 \\
52-141\end{array}$ \\
\hline
\end{tabular}

a Two-dimensional conductivity, defined as the conductance of one square of the $2 \mathrm{D}$ material, i.e., the reciprocal of the sheet resistance. The listed values are conductivities of the FET channel measured at zero gate voltage. 


\section{CONCLUSIONS}

In summary, we have formed graphene on Si(0001) by vacuum annealing, and observed the temperature dependence of the morphology for annealing temperatures in the range of $1100^{\circ} \mathrm{C}$ to $1500^{\circ} \mathrm{C}$. Starting from $\mathrm{H}$-etched substrates that have a regular step-terrace structure, the morphology is found to roughen but maintain the overall step morphology for temperatures of about $1150^{\circ} \mathrm{C}$. For higher temperatures near $1250^{\circ} \mathrm{C}$, the terraces become more irregular in shape, and pits formed at lower temperatures begin to merge and coarsen. At $1350^{\circ} \mathrm{C}$ and above, the surface develops regions of relatively large terraces, separated by narrower regions with step bunches. On the flat terraces, narrow ridges are seen and are attributed to grain boundaries in the graphene. Faint fractal-like patterns are also imaged on the terraces and are argued to arise from atoms at the graphene/SiC interface. Raman spectroscopy displays clear spectra associated with the graphene, and the average graphene thickness derived from the spectra is in reasonable agreement with that obtained from a model based on observed Auger electron spectra.

\section{ACKNOWLEDGEMENTS}

The authors are grateful to W.J. Choyke, R.P. Devaty, W. Hu, K. Oman, N. Srivastava, and Y. Yin for useful discussions and for technical assistance. This work was supported by the National Science Foundation (Grant DMR-0503 748), and by the Defense Advanced Research Projects Agency through a contract administered by the Air Force Research Laboratory (Contract FA8650-08-C-7823). The work is approved by DARPA for public release, distribution unlimited. Use of the Center for Nanoscale Materials at Argonne National Laboratory was supported by the US Department of Energy, Office of Science, Office of Basic Energy Sciences, under Contract No. DE-AC02-06CH11357. Opinions are those of the authors and are not necessarily endorsed by the funding sources.

\section{REFERENCES}

1. K.S. Novoselov, A.K. Geim, S.V. Morozov, D. Jiang, Y. Zhang, S.V. Dubonos, I.V. Grigorieva, and A.A. Firsov, Science 306, 666 (2004). doi:10.1126/science.1102896.

2. W.A. de Heer, C. Berger, X. Wu, P.N. First, E.H. Conrad, X. Li, T. Li, M. Sprinkle, J. Hass, M.L. Sadowski, M. Potemski, and G. Martinez, Solid State Commun. 143, 92 (2007). doi:10.1016/j.ssc.2007.04.023.
3. J. Kedzierski, P.-L. Hsu, P. Healey, P. Wyatt, C.L. Keast, M. Sprinkle, C. Berger, and W.A de Heer, IEEE Trans. Electron. Dev. 55, 2078 (2008). doi:10.1109/TED.2008. 926593.

4. Y.Q. Wu, P.D. Ye, M.A. Capano, Y. Xuan, Y. Sui, M. Qi, J.A. Cooper, T. Shen, D. Pandey, G. Prakash, and R. Reifenberger, Appl. Phys. Lett. 92, 092102 (2008). doi:10.1063/ 1.2889959 .

5. G. Gu, S. Nie, R.M. Feenstra, R.P. Devaty, W.J. Choyke, W.K. Chan, and M.G. Kane, Appl. Phys. Lett. 90, 253507 (2007). doi:10.1063/1.2749839.

6. J.A. Northrup and J. Neugebauer, Phys. Rev. B 57, 4230 (1998). doi:10.1103/PhysRevB.57.R4230.

7. S. Mroczkowski and D. Lichtman, Surf. Sci. 131, 159 (1983). doi:10.1016/0039-6028(83)90125-5.

8. P. Mårtensson, F. Owman, and L.I. Johansson, Phys. Status Solidi 202, 501 (1983). doi:10.1002/1521-3951(199707)202: $1<501::$ AID-PSSB501 > 3.0.CO;2-H.

9. W. Chen, H. Xu, L. Liu, X. Gao, D. Qi, G. Peng, S.C. Tan, Y. Feng, K.P. Loh, and A.T.S. Wee, Surf. Sci. 596, 176 (2005).

10. J.B. Hannon and R.M. Tromp, Phys. Rev. B 77, 241404 (2008). doi:10.1103/PhysRevB.77.241404.

11. V. Ramachandran, M.F. Brady, A.R. Smith, R.M. Feenstra, and D.W. Greve, J. Electron. Mater. 27, 308 (1997). doi:10.1007/s11664-998-0406-7.

12. Although the morphology of Fig. 3a is quite similar to that seen in Ref. 5 , the annealing temperature reported there is higher $\left(1300^{\circ} \mathrm{C}\right)$ and graphene thickness greater (1.5 ML) than the present work. It should be noted however that the starting surface in Ref. 5 is different, since the sample was transferred through air between $\mathrm{H}$-etching and graphitization and also a small amount of surface metal contamination was present. Additionally, some uncertainty in temperature determination occurs in both experiments.

13. I. Forbeaux, J.-M. Themlin, and J.-M. Debever, Phys. Rev. B 58, 16396 (1998). doi:10.1103/PhysRevB.58.16396.

14. C. Riedl, U. Starke, J. Bernhardt, M. Franke, and K. Heinz, Phys. Rev. B 76, 245406 (2007). doi:10.1103/PhysRevB.76. 245406.

15. T.A. Witten and L.M. Sander, Phys. Rev. Lett. 47, 1400 (1981). doi:10.1103/PhysRevLett.47.1400.

16. S.W. Poon, W. Chen, E.S. Tok, and A.T.S. Wee, Appl. Phys. Lett. 92, 104102 (2008). doi:10.1063/1.2883941.

17. S. Nie (Ph.D. Thesis, Department of Physics, Carnegie Mellon University, 2007).

18. A.C. Ferrari, J.C. Meyer, V. Scardaci, C. Casiraghi, M. Lazzeri, F. Mauri, S. Piscanec, D. Jiang, K.S. Novoselov, S. Roth, and A.K. Geim, Phys. Rev. Lett. 97, 187401 (2006). doi:10.1103/PhysRevLett.97.187401.

19. Z.H. Ni, W. Chen, X.F. Fan, J.L. Kuo, T. Yu, A.T.S. Wee, and Z.X. Shen, Phys. Rev. B 77, 115416 (2008). doi:10.1103/Phys RevB.77.115416.

20. J.C. Burton, L. Sun, F.H. Long, Z.C. Feng, and I.T. Ferguson, Phys. Rev. B 59, 7282 (1999). doi:10.1103/PhysRevB.59.7282.

21. D. Graf, F. Molitor, K. Ensslin, C. Stampfer, A. Jungen, C. Hierold, and D. Wirtz, Nano Lett. 7, 238 (2007). doi:10.1021/ nl061702a.

22. G.M. Rutter, N.P. Guisinger, J.N. Crain, E.A.A. Jarvis, M.D. Stiles, T. Li, P.N. First, and J.A. Stroscio, Phys. Rev. B 76, 235416 (2007). doi:10.1103/PhysRevB.76.235416.

23. S. Nie and R.M. Feenstra, J. Vac. Sci. Technol. B, submitted. 